\title{
Actin Filaments Mediate Mechanical Gating during Osmosensory Transduction in Rat Supraoptic Nucleus Neurons
}

\author{
Zizhen Zhang, Alexandra N. Kindrat, Reza Sharif-Naeini, and Charles W. Bourque \\ Centre for Research in Neuroscience, Montreal General Hospital and McGill University, Montreal, Quebec, Canada H3G 1A4
}

\begin{abstract}
Osmosensory transduction is a bidirectional process displayed by neurons involved in the control of thirst and antidiuretic hormone release, and is therefore crucial for body fluid homeostasis. Although this mechanism is known to involve the activation of nonselective cation channels during hypertonicity-evoked shrinking, and the inhibition of these channels during hypotonicity-evoked swelling, the basis for this regulation is unknown. Here, we investigated this process using whole-cell patch-clamp recordings from neurons acutely isolated from the supraoptic nucleus of adult rats. The mechanosensitivity index, defined as the ratio of conductance change to normalized volume change, was quantitatively equivalent whether cell volume was increased or decreased by changes in extracellular fluid osmolality, or by changes in pipette pressure. Moreover, responses induced by hyperosmotic or hypo-osmotic media could be reversed by increasing or decreasing pipette pressure, respectively. The mechanosensitivity index was significantly reduced in neurons treated with cytochalasin-D, a compound that promotes the depolymerization of actin filaments. Conversely, cells treated with jasplakinolide, a compound that promotes actin polymerization, showed a significant increase in mechanosensitivity index. Finally, the depolarizing and excitatory effects of hypertonic stimuli were significantly enhanced by jasplakinolide and reduced by cytochalasin-D. We conclude that osmosensory transduction in these neurons is a reversible mechanical process that depends on an intact actin cytoskeleton, and the sensitivity of the transducer appears to vary in proportion with the density of actin filaments.
\end{abstract}

Key words: hypothalamus; supraoptic nucleus; electrophysiology; osmoreceptor; actin cytoskeleton; vasopressin

\section{Introduction}

Osmosensory neurons in the CNS of mammals detect changes in plasma osmolality caused by temporal differences in the intake or loss of salt and water. In vivo, the electrical activity of these neurons is generally increased by hyperosmolality and inhibited by hypo-osmolality (Bourque et al., 1994), and information encoded by these responses modulates thirst and antidiuretic hormone release to promote hydromineral balance (Denton et al., 1996). Previous studies have shown that osmosensory transduction in magnocellular neurosecretory cells (MNCs) of the rat supraoptic nucleus is mediated by the modulation of $\mathrm{Gd}^{3+}$ sensitive, stretch-inhibited cation (SIC) channels during osmotically evoked changes in cell volume (Oliet and Bourque, 1993a,b, 1996). Specifically, hypertonic stimuli depolarize and excite MNCs as a result of increased SIC channel activity during cell shrinking, whereas the cells are hyperpolarized and inhibited be-

Received July 31, 2006; revised March 5, 2007; accepted March 6, 2007

This work was supported by Canadian Institutes of Health Research (CIHR) Operating and Senior Investigator Awards and by a James McGill Research Chair to C.W.B. In addition, A.N.K. was the recipient of studentship awards from the Fonds pour la Formation de Chercheurs et I'Aide à la Recherche of Quebec and from the McGill University Health Center Research Institute (MUHCRI). R.S.-N. was the recipient of doctoral awards from the CIHR and the Heart and Stroke Foundation of Canada (HSFC), and Z.Z. was the recipient of studentship awards from the MUHCRI and the HSFC.

Correspondence should be addressed to Dr. Charles W. Bourque, Division of Neurology, Room L7-216, Montreal General Hospital, 1650 Cedar Avenue, Montreal, Quebec, Canada H3G 1A4. E-mail: charles.bourque@mcgill.ca.

D0I:10.1523/JNEUROSCI.3278-06.2007

Copyright $\odot 2007$ Society for Neuroscience $\quad$ 0270-6474/07/274008-06\$15.00/0 cause of reduced SIC channel activity in response to hypotonicity-evoked cell swelling (Voisin and Bourque, 2002). Although single SIC channels recorded in cell-attached membrane patches display an intrinsic mechanosensitivity that is consistent with the macroscopic responses observed during osmosensory transduction (i.e., stretch-inhibition) (Oliet and Bourque, 1993a, 1996), it is not known whether the modulation of these channels during whole-cell recordings from osmotically stimulated MNCs is caused by the mechanosensitive properties of the channels, or to other forms of regulation. Indeed, the apparent mechanosensitivity of ion channels can be exaggerated by the patch-clamp recording technique (Morris and Horn, 1991; Hamill and McBride, 1997) and osmotic modulation of ion channels can be mediated by other forms of regulation (Strange et al., 1996; Nilius et al., 1997; Wehner et al., 2003; Shukla et al., 2004). Notably, previous studies have shown that changes in ionic strength, rather than cell volume, mediate the osmotic modulation of volumeregulated (Nilius et al., 1998; Voets et al., 1999) and swellingactivated anion channels (Cannon et al., 1998) in various cells. It is therefore of fundamental importance to establish whether the osmotic modulation of native osmosensory neurons depends on changes in ionic strength, or on a mechanical stimulus associated with osmotically evoked changes in cell volume.

\section{Materials and Methods}

Preparation and perfusion of isolated cells. MNCs were acutely isolated from adult (100-200 g) male Long-Evans rats as reported previously 

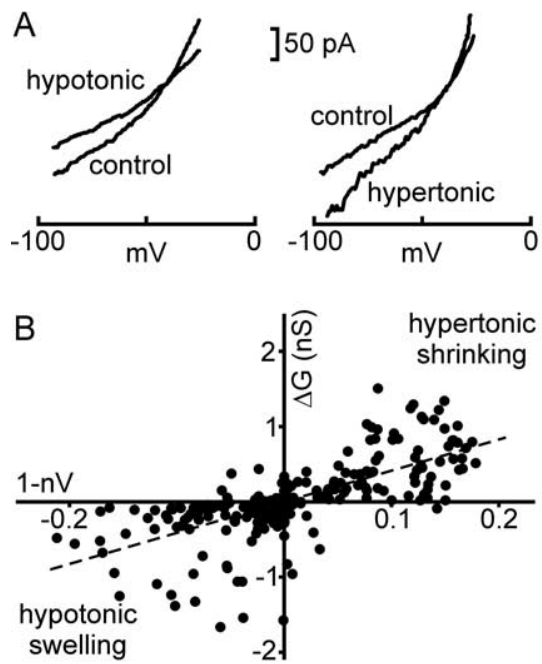

Figure 1. Volume modulation of cation current by osmotic and hydrostatic pressure. $\boldsymbol{A}$, Steady-state $/-V$ relationships measured from isolated MNCs $\left(V_{H}=-70 \mathrm{mV}\right.$ ) exposed to hypotonic (left) and hypertonic solutions (right). Note that the stimuli respectively reduce and increase slope conductance. $\boldsymbol{B}$, Relationships between changes in slope conductance $(\Delta G)$ and volume decrease $(1-n V)$ during osmotic stimulation. Each point depicts simultaneous measures of $\Delta G$ and $1-n V$ taken at several time points during hypotonic (bottom left quadrant) or hypertonic stimuli (top right quadrant). Solid line is a linear regression fit of the data (slope $=4.1 \mathrm{nS} / \mathrm{n} \Delta V ; n=22 ; r=0.6847$ ). C, Steady-state $I-V$ relationships measured from cells inflated by positive pressure in the patch pipette (left) and shrunken by negative pressure (right). Note that the stimuli respectively reduce and increase slope conductance. $D$, Relationship between $\Delta G$ and $1-n V$ during pressure-evoked responses. Points depict simultaneous measures of $\Delta G$ and $1-n V$ taken at various times during application of positive pressure (bottom left quadrant) or negative pressure (top right quadrant). The solid line is a linear regression fit of the data (slope $=3.9 \mathrm{nS} / \mathrm{n} \Delta V ; r=0.602 ; n=21$ ). Mean values of the reversal potentials of the ionic currents modulated by hypo-0smolality $(-36 \pm 3 \mathrm{mV})$, hyperosmolality $(-31 \pm 4 \mathrm{mV})$, positive pressure $(-33 \pm 5 \mathrm{mV})$, and negative pressure $(-33 \pm 4 \mathrm{mV})$ were not significantly different from one another ( $p=0.83$; one-way ANOVA), and were consistent with the involvement of SIC channels in all of these responses. The slopes of the regression lines shown in $\boldsymbol{B}$ and $\boldsymbol{D}$ are not different ( $p>0.05$ ).

(Zhang and Bourque, 2003). Briefly, brain slices containing the supraoptic nucleus (SON) were cut and placed in oxygenated $\left(100 \% \mathrm{O}_{2}\right)$ piperazine- $N, N^{\prime}$-bis[2-ethanesulfonic acid] (PIPES)-buffered saline, $\mathrm{pH}$ $7.4\left(22^{\circ} \mathrm{C}\right.$ ), containing (in mM) $120 \mathrm{NaCl}, 3 \mathrm{KCl}, 1 \mathrm{MgCl}_{2}, 1 \mathrm{CaCl}_{2}, 25$ dextrose, and 10 PIPES. Blocks $\left(\sim 1 \mathrm{~mm}^{3}\right)$ containing the SON were then cut and incubated for $90 \mathrm{~min}$ in oxygenated PIPES solution $\left(33.5^{\circ} \mathrm{C}\right)$, $\mathrm{pH} 7.0$, containing $0.7 \mathrm{mg} / \mathrm{ml}$ trypsin (type XI; Sigma, St. Louis, MO). Blocks were transferred to a trypsin-free PIPES solution, $\mathrm{pH} 7.4\left(22^{\circ} \mathrm{C}\right)$, and dispersed as required. Cell suspensions were plated onto plastic Petri dishes and used within 30-120 min. Dishes were mounted on an inverted phase contrast microscope (Diaphot; Nikon, Tokyo, Japan) and perfused with a HEPES-buffered saline containing (in mM) $75 \mathrm{Na}_{2} \mathrm{SO}_{4}, 3 \mathrm{KCl}, 1$ $\mathrm{MgCl}_{2}, 10 \mathrm{HEPES}, 1 \mathrm{CaCl}_{2}$, and 10 glucose. Different amounts of mannitol were added to adjust osmolality. Osmotic steps were applied using a gravity system delivering solutions at a rate of $1.0-1.2 \mathrm{ml} / \mathrm{min}^{-1}$. A fast stepper device (SF-77B; Warner Instruments, Hamden, CT) was used to switch between the adjacent barrels of a three-barrel glass assembly to minimize time lag $(<20 \mathrm{~ms})$ when changing solutions. Excess solution was sucked away through a vacuum system.

Measurements of relative cell volume. We determined relative changes in cell volume from measurements of maximal cross-sectional area (CSA; from digitized images) (Zhang and Bourque, 2003). The perimeter of the cell was traced using Scion (Frederick, MD) Image for Windows 4.02 and the CSA (in pixels) was determined by the software. All values of CSA during control were averaged $\left(C S A_{\mathrm{o}}\right)$ and values of relative (normalized) volume at each time point $\left(\mathrm{n} V_{\mathrm{t}}\right)$ were calculated from the CSA value at that time point $\left(C S A_{\mathrm{t}}\right)$ using the equation; $\mathrm{n} V_{\mathrm{t}}=\left[\left(C S A_{\mathrm{t}}\right)^{1.5} /\right.$ $\left.\left(C S A_{\mathrm{o}}\right)^{1.5}\right]$. This formula assumes that cells are simple ellipsoids and that changes in volume occur uniformly in all planes. Cells with the simplest ellipsoid morphologies and shortest processes were thus selected for analysis.

Electrophysiological recording. Cells were patch clamped with glass pipettes (1.2 mm outer diameter glass; A-M Systems, Carlsborg, WA) con-

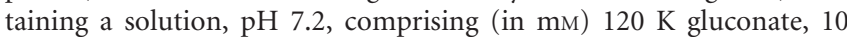

HEPES, $1 \mathrm{MgCl}_{2}$, and 1 EGTA. Whole-cell recordings were performed using an Axopatch-1D amplifier (Molecular Devices, Union City, CA). Pipette pressure was monitored via a digital pressure transducer (model PM015D; World Precision Instruments, Sarasota, FL) and controlled via an air-filled syringe. Changes in volume caused by given changes in pipette pressure differed significantly from cell to cell and, generally, varied as a sensitive and inverse function of pipette resistance (i.e., tip diameter). Membrane current (direct current, $-2 \mathrm{kHz})$ was digitized $(10 \mathrm{kHz})$ via a digidata 1200B interface coupled to a computer running Clampex 8 (Molecular Devices). Wholecell capacitance and series resistance were compensated electronically. Voltage ramps $(-100-0$ $\mathrm{mV} ; 20 \mathrm{mV} / \mathrm{s}$ ) were commanded to determine steady-state whole-cell current-voltage $(I-V)$ relationships. All data were stored on a computer for off-line analysis.

Treatment of cells with JSK and Cyt-D. Stock solutions of cytochasin D (Cyt-D) (78-100 mM in DMSO; Sigma) or jasplakinolide (JSK; $1 \mathrm{~mm}$ in DMSO; Invitrogen, Eugene, OR) were separated into $1 \mu \mathrm{l}$ aliquots and stored at $-20^{\circ} \mathrm{C}$ in plastic vials. For each experiment, a fresh $1 \mu \mathrm{l}$ aliquot of drug or DMSO was well mixed into $400 \mu \mathrm{l}$ of oxygenated PIPES solution, yielding concentrations of 195-250 $\mu \mathrm{M}$ Cyt-D and 2.5 $\mu \mathrm{M}$ JSK in $0.25 \%$ DMSO. Enzyme-treated tissue blocks were then placed and triturated in this solution and plated onto 4-5 plastic Petri dishes. Dishes containing drug treated cells (30-60 min after plating) were then flooded with HEPES recording solution just before recording was to begin.

Statistics. All values are reported as mean \pm SEM. Comparisons of the means between groups were made using Student's $t$ test or ANOVA (SigmaStat 2.1; SPSS, Chicago, IL) as indicated. Differences between the means were considered significant when $p<0.05$. Where differences were found by ANOVA and Dunn's test for multiple comparisons was performed post hoc to identify specific distinctions $(p<0.05)$.

\section{Results}

If osmosensory transduction is attributable primarily to a mechanical regulation of SIC channels, then this process should be quantitatively equivalent when induced by changes in cell volume provoked by osmotic perturbations and by changes in hydrostatic pressure applied under isotonic conditions. To test this hypothesis, whole-cell voltage-clamp recordings were made from MNCs acutely isolated from the supraoptic nucleus of adult rats. Cells were challenged with osmotic stimuli (in the absence or presence of mannitol) or changes in hydrostatic pressure (applied to the inside of the recording pipette), and synchronous values of normalized cell volume $(\mathrm{n} V)$ and membrane conductance were determined at different time points by digital imaging and steady-state $I-V$ analysis. As reported previously (Oliet and Bourque, 1993a), MNCs challenged with hypotonic stimuli showed decreases in membrane conductance, whereas those challenged with hypertonicity showed increases in membrane conductance (Fig. 1 A). Pooled data obtained from 22 osmotically challenged cells confirmed that changes in membrane conductance $(\Delta G)$ vary inversely with cell volume. To obtain a positive numerical index of transducer sensitivity, values of $\Delta G$ were plotted as a function of the corresponding normalized volume decrease ( $\mathrm{n} \Delta V$; i.e., $1-\mathrm{n} V$ ) observed at various time points, and linear regression analysis was performed (Fig. $1 B$ ). The slope of 

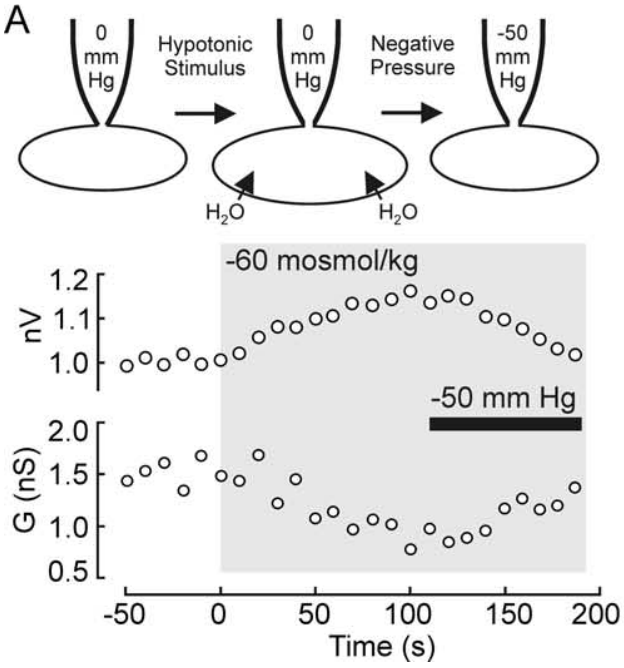
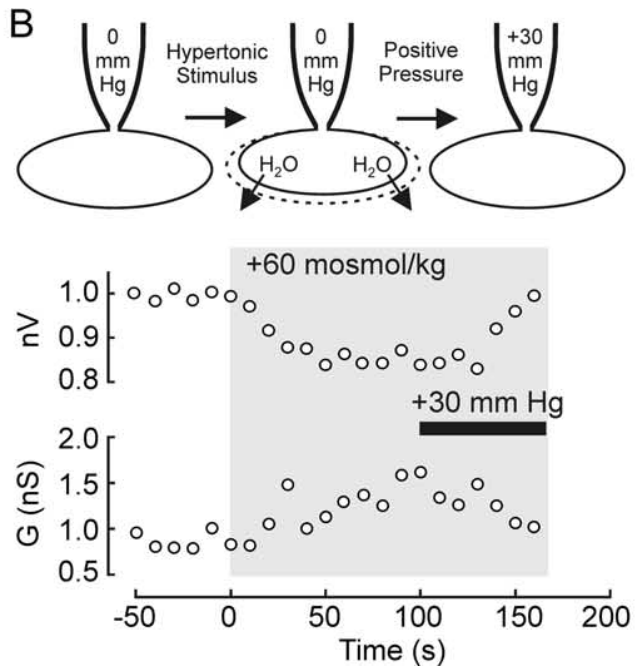

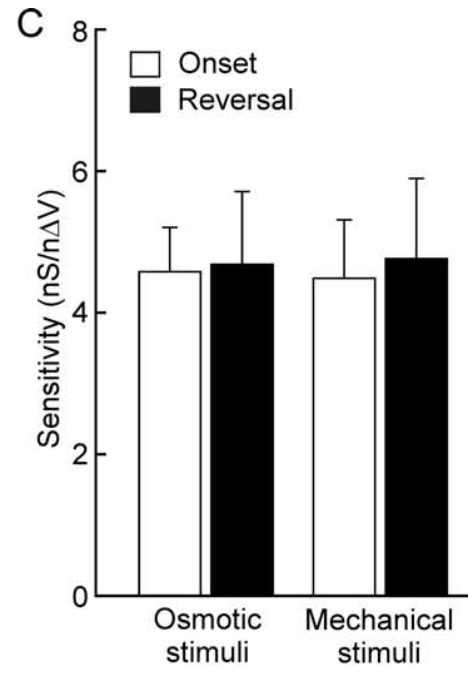

Figure 2. Reversal of osmosensory transduction by hydrostatic pressure. $A$, Plot showing values of membrane conductance $(G)$ and $\mathrm{n} V$ observed in an isolated MNC exposed to a sustained hypotonic stimulus ( $-60 \mathrm{mosmol} / \mathrm{kg}$, gray area). After $110 \mathrm{~s}$, negative pressure (bar, $-50 \mathrm{mmHg}$ ) was applied to the recording pipette. Note that this procedure reversed the effects of the hypotonic stimulus on $\mathrm{nV}$ and $G$. B. Plot showing values of $G$ and $\mathrm{nV}$ observed in a cell exposed to a sustained hypertonic stimulus (+60 mosmol/kg, gray area). After $100 \mathrm{~s}$, positive pressure (bar, $+30 \mathrm{mmHg}$ ) was applied to the recording pipette. Note that this procedure reversed the effects of the hypertonic stimulus on $n V$ and $G$. C, Bar graphs showing mean values of transducer sensitivity observed when hydrostatic pressure and osmotic stimuli were used to initiate (onset, open bars) or reverse (reversal, filled bar) the modulation of membrane cation conductance by changes in cell volume. No significant difference was observed ( $p>0.05$; two-way ANOVA).

this regression $(4.1 \mathrm{nS} / \mathrm{n} \Delta V ; n=22 ; r=0.68)$ was taken as a measurement of the sensitivity of the relationship between SIC channel activity and changes in cell volume during osmosensory transduction. A distinct set of MNCs $(n=21)$ were then subjected to changes in cell volume induced by application of positive or negative pressure to the recording pipette. As shown in Figure $1 C$, positive pressure caused a decrease in membrane conductance whereas reducing pipette pressure provoked an increase in membrane conductance. Regression analysis of pooled data (Fig. $1 D$ ) revealed that values of $\Delta G$ varied as a linear function of $\mathrm{n} \Delta V$ with a slope of $3.9 \mathrm{nS} / \mathrm{n} \Delta V$; $r=0.60 ; n=21$ ), a value not significantly different from that obtained during osmotic stimulation $(p>0.05)$. These results indicate that volume-dependent regulation of SIC channel activity is quantitatively equivalent during osmotic and mechanical stimulation.

If the mechanical gating of SIC channels is solely responsible for osmosensory transduction, then it should be possible to interrupt and reverse the osmotic modulation of the channels by a mechanically induced recovery of cell volume. Indeed, the decrease in membrane conductance associated with hypotonic swelling could be reversed by reducing cell volume via the application of negative pressure to the recording pipette (Fig. $2 \mathrm{~A}$ ). Conversely, the increase in membrane conductance provoked by hypertonic shrinking could be reversed by inflating the cell via an increase in pipette pressure (Fig. $2 \mathrm{~B}$ ). We also attempted to reverse the effects of mechanically evoked changes in cell volume by antagonistic osmotic stimuli. Whereas we could readily reverse suction-evoked increases in conductance by exposing the cell to a hypotonic solution $(n=6)$ (data not shown), we could not maintain stable whole-cell recordings long enough to examine the effects of hypertonicity on the reduced conductance provoked during sustained increases in pipette pressure. Linear regression analysis of data obtained from individual cells was then performed to determine the slope of the relationship between $\Delta G$ and $\mathrm{n} \Delta V$ when osmotic and mechanical stimuli were used to initiate, or reverse, the modulation of SIC channels. As illustrated in Figure $2 C$, the sensitivity of the transducer was statistically equivalent under all conditions $(p>0.05)$. Together, these findings indicate that osmosensory transduction relies primarily on a reversible mechanical modulation of SIC channels during osmotically evoked changes in cell volume.

Previous studies have indicated that filamentous actin (Factin) can either participate in the gating of mechanosensitive channels (Su et al., 2000; Cho et al., 2002; Piao et al., 2003) or protect against their activation by mechanical stimuli by providing physical support for the plasma membrane (Hamill and McBride, 1997; Ko and McCulloch, 2000; Morris, 2001). To determine whether $\mathrm{F}$-actin is required for mechanical regulation of SIC channels, we quantified transducer sensitivity in MNCs pretreated (30-60 min) with Cyt-D (200-250 $\mu \mathrm{M})$, a drug that depolymerizes F-actin (Cooper, 1987), or JSK (2.5 $\mu \mathrm{M})$, a drug that promotes actin polymerization (Bubb et al., 1994). Values of $\Delta G$ and $\mathrm{n} \Delta V$ were monitored simultaneously at regular intervals while a decrease in cell volume was provoked by applying negative pressure through the recording pipette for a period of $60 \mathrm{~s}$. Interestingly, only a few cells showed a complete recovery of cell volume after termination of the stimulus. Thus, to ensure that the responses observed reflected the activation of SIC channels rather than a disruption of the seal between cell and pipette, only recordings in which the suction-induced current could be terminated by application of $250 \mu \mathrm{M} \mathrm{Gd}{ }^{3+}$ were retained for analysis. As illustrated in Figure $3 A$, MNCs treated with Cyt-D showed a smaller inward current response to suction compared with controls, whereas those treated with JSK displayed a larger response to the stimulus. Indeed, the mean transducer sensitivity index was significantly lower in cells treated with Cyt-D $(1.1 \pm 0.5 \mathrm{nS} / \mathrm{n} \Delta V ; n=21)$ and higher in cells treated with JSK $(19.1 \pm 3.1 \mathrm{nS} / \mathrm{n} \Delta V ; n=22 ; p<0.05)$ compared with control MNCs $(4.1 \pm 0.5 \mathrm{nS} / \mathrm{n} \Delta V ; n=41)$ (Fig. $3 B$ ). These results indicate that $\mathrm{F}$-actin is necessary for the volumedependent modulation of SIC channels, and that the mechanosensitivity of MNCs varies in proportion with the amount of F-actin that is present in these cells. 

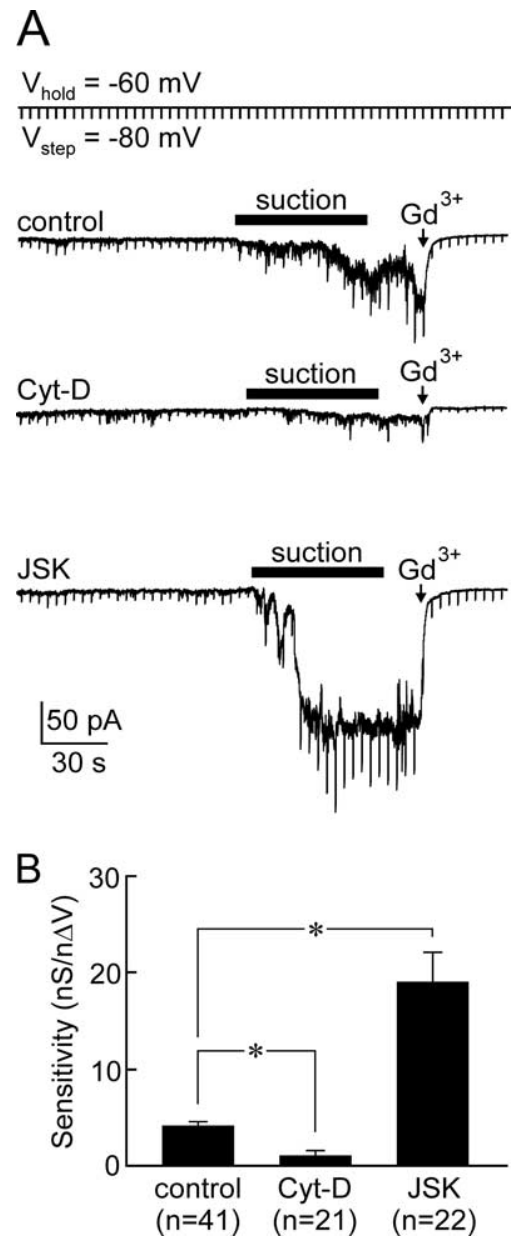

Figure 3. Role of F-actin in mechanotransduction. $A$, Whole-cell current recordings $\left(V_{\mathrm{H}}=\right.$ $-60 \mathrm{mV}$ ) from isolated MNCs exposed to Cyt-D (middle), vehicle (control, top), or JSK (bottom). Vertical deflections are current responses to hyperpolarizing steps $(-20 \mathrm{mV})$ applied every $5 \mathrm{~s}$ to monitor $G$. In each case, negative pressure $(\sim-100 \mathrm{mmHg}$ ) was applied to the patch pipette (bar) to induce a decrease in cell volume of $\sim 10 \%$. Note that $\mathrm{Gd}^{3+}(250 \mu \mathrm{m})$ was applied near the end of each recording to confirm that the current induced was caused by the activation of SIC channels. $\boldsymbol{B}$, Bar graph showing mean ( \pm SEM) values of transducer mechanosensitivity measured in each group $\left({ }^{*} p<0.05 ;\right.$ ANOVA).

Finally, to determine whether F-actin is necessary for osmosensory transduction, we examined the effects of Cyt-D and JSK on the current-clamp responses of MNCs to a hyperosmotic challenge (+60 mosmol/kg; 60-80 s). As illustrated in Figure 4A, excitatory responses to this stimulus were smaller in Cyt-D treated cells $(n=20)$ and larger in JSK treated cells $(n=11)$ than in vehicle-treated controls $(n=15)$. Indeed, although osmotically evoked changes in cell volume were equivalent in the three groups (Fig. $4 B)(p=0.973)$, significant differences were observed in the magnitude of the depolarizing osmoreceptor potentials and excitatory responses observed under the three conditions. Specifically, Cyt-D significantly reduced the average amplitude of the osmoreceptor potentials $(0.6 \pm 0.8 \mathrm{mV}$ in Cyt-D vs $8.1 \pm 1.3 \mathrm{mV}$ in control; $p<0.001)$ and the increase in firing rate $(+0.3 \pm 0.2 \mathrm{~Hz}$ in Cyt-D vs $+1.6 \pm 0.5 \mathrm{~Hz}$ in control; $p<0.05)$ induced by the hyperosmotic stimulus. Conversely, JSK significantly enhanced both the osmoreceptor potential $(+12.5 \pm 1.4 \mathrm{mV} ; p=0.013)$ and the excitatory effect $(+3.1 \pm$ $0.7 \mathrm{~Hz} ; p=0.012$ ) of the hyperosmotic stimulus.
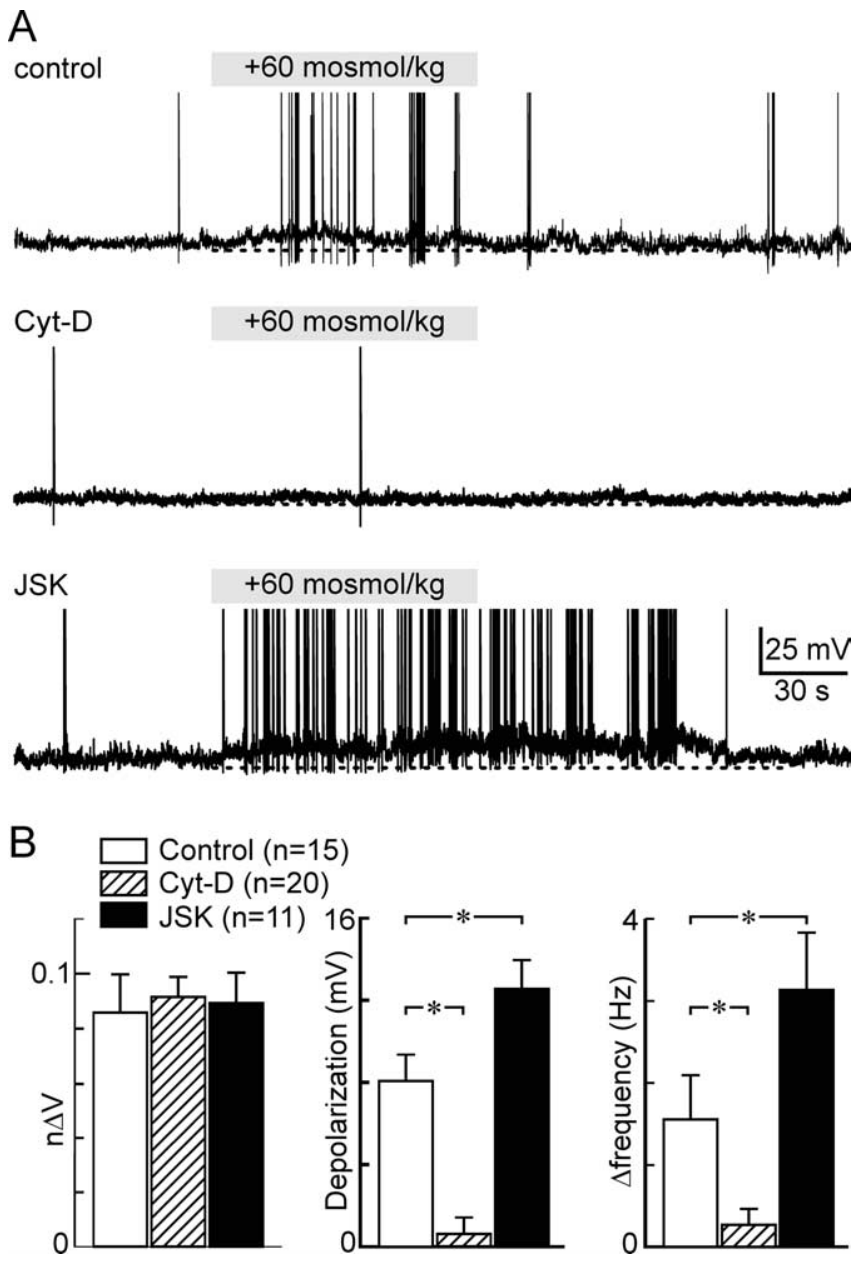

Figure 4. F-actin is required for osmosensory transduction. $\boldsymbol{A}$, Whole-cell current-clamp recordings from isolated MNCs subjected to hypertonic stimuli (bar; $+60 \mathrm{mosmol} / \mathrm{kg}$ ). The traces show representative recordings taken from cells treated with vehicle (control, top), Cyt-D (middle), and JSK (bottom). In all traces, the spikes are truncated and the dashed line indicates $-55 \mathrm{mV}$. B, Bar graphs showing that mean ( \pm SEM) osmoreceptor potentials (depolarization measured at $60 \mathrm{~s}$; middle) and excitation (increase in firing observed between 50 and $70 \mathrm{~s}$ compared with control; right) were significantly reduced by (yt-D and significantly enhanced by JSK, whereas no difference was noted in the amount of shrinking evoked by the osmotic stimulus ( $p=0.923$; measured 60 s after stimulus onset; left). ${ }^{*} p<0.05$.

\section{Discussion}

Osmosensory transduction is a mechanical event

Our experiments revealed that the sensitivity of the relationship between cell volume and membrane cation conductance was quantitatively equivalent whether the volume of MNCs was modulated by osmotic stimuli, or by changes in pipette pressure applied under isotonic conditions. Although the maximal amplitudes of the stimuli used in our experiments provoked changes in cell volume that exceeded those that occur under physiological conditions, it is important to note that the inverse relationship between membrane conductance and cell volume could be clearly detected with volume changes of $2-3 \%$ (Fig. $1 B$ ), well within the physiological range (Zhang and Bourque, 2003). Moreover, the modulation of SIC channels provoked by hypotonic or hypertonic stimuli could be reversed by inducing a recovery of cell volume by applying opposite changes in hydrostatic pressure to the recording pipette. In these experiments, reversal of the transduction current occurred despite the continued presence of the changes in ionic strength that accompanied superim- 
posed osmotic stimuli. These observations indicate that osmosensory transduction in MNCs is triggered specifically by changes in the volume of the neurons, and not by changes in ionic strength. Thus, osmosensory transduction is primarily a mechanical event.

\section{Actin filaments mediate mechanical coupling during osmosensory transduction}

Previous studies have established that elements of the cytoskeleton are attached to the plasma membrane via links to a variety of membrane-associated proteins (Revenu et al., 2004), and that the cross-linked fibers that constitute the submembrane and cytoplasmic components of the cytoskeleton can distribute forces applied on one part of the cell surface to other parts of the cell via tensegrity (Ingber, 1997). The cytoskeleton therefore represents a potentially strategic component of the osmoreceptor complex that could relay forces generated during changes in cell volume to the molecular mechanotransduction elements that modulate SIC channel activity (i.e., accessory proteins or the channel itself). Indeed, mechanically evoked increases in cation current and excitatory responses to hypertonicity were both blunted by Cyt-D, a compound that promotes actin depolymerization (Cooper, 1987), and enhanced in MNCs treated with JSK, a compound that promotes the accumulation of F-actin (Bubb et al., 1994). In addition to revealing that actin filaments play a key role in the osmotic and mechanical regulation of SIC channels, these findings suggest that the sensitivity of the osmosensory transducer varies in proportion with the amount of $\mathrm{F}$-actin present in MNCs. This hypothesis is consistent with previous studies showing that the stiffness of a cross-linked actin network increases as a function of the concentration of F-actin (Gardel et al., 2004; Shin et al., 2004). Accordingly, if an elastic network of cross-linked F-actin was involved in relaying volumedependent mechanical forces to the transducer element, then the amount of force applied per unit volume change should increase with F-actin density and, thus, result in an enhancement of transducer sensitivity, as was observed in our experiments. Because actin polymerization and depolymerization can be regulated through receptor-mediated signaling cascades (Cicchetti et al., 2002; Revenu et al., 2004), it is conceivable that neurotransmitter-mediated changes in actin density could underlie the modulation of homeostatic osmoregulatory reflex gain that has been observed under various physiological and pathological conditions (Robertson et al., 1976; De Smet et al., 2003).

\section{Molecular basis for mechanical control of SIC channels during osmosensory transduction}

The molecular mechanism that underlies the modulation of SIC channels during osmosensory transduction remains unknown and may involve proteins other than F-actin and the SIC channel itself. Although our results cannot exclude the possibility that an actin-dependent mechanically gated biochemical step is involved in the osmotic modulation of SIC channels, such a process would have to be bidirectional, and rapidly reversible, because the time course of mechanotransduction mirrored that of the changes in cell volume. Alternately, it remains possible that volumedependent modulation of SIC channel activity reflects the intrinsic mechanosensitivity of these channels (Oliet and Bourque, 1993a). In this case, the actin cytoskeleton might mediate volume-dependent force transmission and mechanical gating through direct or indirect physical links with SIC channels (Hamill and Martinac, 2001; Kung, 2005), or it might serve to isolate small domains in the plasma membrane (Ritchie and Kusumi, 2004; McMahon and Gallop, 2005), where unattached SIC channels could sense changes in the local tension or curvature of the lipid bilayer associated with changes in cell volume (Kung, 2005). Finally, it is important to emphasize that our experiments were performed on acutely isolated neurons and that additional factors could significantly contribute to osmosensory transduction in situ. Indeed, it is quite possible that extracellular matrix proteins participate in the mechanical regulation of SIC channels either through a physical association with the cortical cytoskeleton, or by contributing to the overall elasticity of the tissue. Additional studies will be required to examine these possibilities.

\section{References}

Bourque CW, Oliet SH, Richard D (1994) Osmoreceptors, osmoreception, and osmoregulation. Front Neuroendocrinol 15:231-274.

Bubb MR, Senderowicz AM, Sausville EA, Duncan KL, Korn ED (1994) Jasplakinolide, a cytotoxic natural product, induces actin polymerization and competitively inhibits the binding of phalloidin to F-actin. J Biol Chem 269:14869-14871.

Cannon CL, Basavappa S, Strange K (1998) Intracellular ionic strength regulates the volume sensitivity of a swelling-activated anion channel. Am J Physiol 275:C416-C422.

Cho H, Shin J, Shin CY, Lee SY, Oh U (2002) Mechanosensitive ion channels in cultured sensory neurons of neonatal rats. J Neurosci 22:1238-1247.

Cicchetti G, Allen PG, Glogauer M (2002) Chemotactic signaling pathways in neutrophils: from receptor to actin assembly. Crit Rev Oral Biol Med 13:220-228.

Cooper JA (1987) Effects of cytochalasin and phalloidin on actin. J Cell Biol 105:1473-1478.

Denton DA, McKinley MJ, Weisinger RS (1996) Hypothalamic integration of body fluid regulation. Proc Natl Acad Sci USA 93:7397-7404.

De Smet HR, Menadue MF, Oliver JR, Phillips PA (2003) Increased thirst and vasopressin secretion after myocardial infarction in rats. Am J Physiol 285:R1203-R1211.

Gardel ML, Shin JH, MacKintosh FC, Mahadevan L, Matsudaira P, Weitz DA (2004) Elastic behavior of cross-linked and bundled actin networks. Science 304:1301-1305.

Hamill OP, Martinac B (2001) Molecular basis of mechanotransduction in living cells. Physiol Rev 81:685-740.

Hamill OP, McBride Jr DW (1997) Induced membrane hypo/hyper-mechanosensitivity: a limitation of patch-clamp recording. Annu Rev Physiol 59:621-631.

Ingber DE (1997) Tensegrity: the architectural basis of cellular mechanotransduction. Annu Rev Physiol 59:575-599.

Ko KS, McCulloch CA (2000) Partners in protection: interdependence of cytoskeleton and plasma membrane in adaptations to applied forces. J Membr Biol 174:85-95.

Kung C (2005) A possible unifying principle for mechanosensation. Nature 436:647-654

McMahon HT, Gallop JL (2005) Membrane curvature and mechanisms of dynamic cell membrane remodelling. Nature 438:590-596.

Morris CE (2001) Mechanoprotection of the plasma membrane in neurons and other non-erythroid cells by the spectrin-based membrane skeleton. Cell Mol Biol Lett 6:703-720.

Morris CE, Horn R (1991) Failure to elicit neuronal macroscopic mechanosensitive currents anticipated by single-channel studies. Science 251:1246-1249.

Nilius B, Eggermont J, Voets T, Buyse G, Manolopoulos V, Droogmans G (1997) Properties of volume-regulated anion channels in mammalian cells. Prog Biophys Mol Biol 68:69-119.

Nilius B, Prenen J, Voets T, Eggermont J, Droogmans G (1998) Activation of volume-regulated chloride currents by reduction of intracellular ionic strength in bovine endothelial cells. J Physiol (Lond) 506:353-361.

Oliet SH, Bourque CW (1993a) Mechanosensitive channels transduce osmosensitivity in supraoptic neurons. Nature 364:341-343.

Oliet SH, Bourque CW (1993b) Steady-state osmotic modulation of cationic conductance in neurons of the rat supraoptic nucleus. Am J Physiol 265:R1475-R1479. 
Oliet SH, Bourque CW (1996) Gadolinium uncouples mechanical detection and osmoreceptor potential in supraoptic neurons. Neuron 16:175-181.

Piao L, Ho WK, Earm YE (2003) Actin filaments regulate the stretch sensitivity of large-conductance, $\mathrm{Ca}^{2+}$-activated $\mathrm{K}^{+}$channels in coronary artery smooth muscle cells. Eur J Physiol 446:523-528.

Revenu C, Athman R, Robine S, Louvard D (2004) The co-workers of actin filaments: from cell structures to signals. Nat Rev Mol Cell Biol 5:635-646.

Ritchie K, Kusumi A (2004) Role of the membrane skeleton in creation of microdomains. Subcell Biochem 37:233-245.

Robertson GL, Shelton RL, Athar S (1976) The osmoregulation of vasopressin. Kidney Int 1:25-37.

Shin JH, Gardel ML, Mahadevan L, Matsudaira P, Weitz DA (2004) Relating microstructure to rheology of a bundled and cross-linked F-actin network in vitro. Proc Natl Acad Sci USA 101:9636-9641.

Shukla A, Hashiguchi N, Chen Y, Coimbra R, Hoyt DB, Junger WG (2004)
Osmotic regulation of cell function and possible clinical applications. Shock 21:391-400.

Strange K, Emma F, Jackson PS (1996) Cellular and molecular physiology of volume-sensitive anion channels. Am J Physiol 270:C711-C730.

Su X, Wachtel RE, Gebhart GF (2000) Mechanosensitive potassium channels in rat colon sensory neurons. J Neurophysiol 84:836-843.

Voets T, Droogmans G, Raskin G, Eggermont J, Nilius B (1999) Reduced intracellular ionic strength as the initial trigger for activation of endothelial volume-regulated anion channels. Proc Natl Acad Sci USA 96:5298-5303.

Voisin DL, Bourque CW (2002) Integration of sodium and osmosensory signals in vasopressin neurons. Trends Neurosci 25:199-205.

Wehner F, Olsen H, Tinel H, Kinne-Saffran E, Kinne RK (2003) Cell volume regulation: osmolytes, osmolyte transport, and signal transduction. Rev Physiol Biochem Pharmacol 148:1-80.

Zhang Z, Bourque CW (2003) Osmometry in osmosensory neurons. Nat Neurosci 6:1021-1022. 